\title{
ON BOUNDS FOR THE DIFFERENCE BETWEEN THE STOP-LOSS TRANSFORMS OF TWO COMPOUND DISTRIBUTIONS
}

\author{
BJøRN SUNDT ${ }^{1}$ AND J AN DHAENE $^{2}$
}

\begin{abstract}
In the present note we deduce a class of bounds for the difference between the stoploss transforms of two compound distributions with the same severity distribution. The class contains bounds of any degree of accuracy in the sense that the bounds can be chosen as close to the exact value as desired; the time required to compute the bounds increases with the accuracy.
\end{abstract}

\section{INTRODUCTION}

During the last twenty years, there has grown up a large literature on approximations and inequalities for stop-loss premiums under various assumptions. One way of approximation is to approximate the original distribution with another distribution that makes the evaluation simpler. In such cases it is useful to have bounds for the difference between the exact stop-loss premium and the approximation, that is, it is of interest to have bounds for the difference between the stop-loss transforms of two distributions.

When approximating the stop-loss transform of a compound distribution, it is sometimes convenient to replace the counting distribution with another distribution, e.g. a Bernoulli distribution or a Poisson distribution, and keep the severity distribution unchanged. Such approximations are discussed by i.a. Dhaene \& Sundt (1996).

In the present note we deduce classes of bounds for the difference between the stoploss transforms of two compound distributions with the same severity distribution. The classes contain bounds of any degree of accuracy in the sense that the bounds can be chosen as close to the exact value as desired; the time required to compute the bounds increases with the accuracy.

\section{NOTATION AND CONVENTIONS}

Let $R_{+}$and $Z_{+}$denote the sets of respectively the non-negative real numbers and the non-negative integers, and $P_{R_{+}}$and $P_{Z_{+}}$the classes of probability distributions with

\footnotetext{
${ }^{1}$ University of Bergen, Norway

${ }^{2}$ Katholieke Universiteit Leuven, Belgium

ASTIN BULLETIN, Vol. 26, No. 2, 1996, pp. 225-231
} 
finite mean on respectively $R_{+}$and $Z_{+}$. For distributions in $P_{R_{+}}$, we shall denote the cumulative distribution function with a capital letter and, for distributions in $P_{Z_{+}}$, the discrete density function with the corresponding lower case letter. The stop-loss transform of a distribution will be denoted by a horizontal bar on the top of the symbol of the distribution, that is, for a distribution $F \in P_{R_{+}}$, we have the stop-loss transform $\bar{F}$ given by

$$
\bar{F}(x)=\int_{x}^{\infty}(y-x) d F(y)=\int_{x}^{\infty}(1-F(y)) d y . \quad(x \geq 0)
$$

The mean of $F$ is denoted by $\mu_{F}$, that is,

$$
\mu_{F}=\bar{F}(0)=\int_{0}^{\infty} y d F(y)=\int_{0}^{\infty}(1-F(y)) d y .
$$

We shall denote a compound distribution with counting distribution $P \in P_{Z_{+}}$and severity distribution $H \in P_{\mathbb{R}_{+}}$by $P \vee H$, that is,

$$
P \vee H=\sum_{n=0}^{\infty} p(n) H^{n *},
$$

where $H^{n^{*}}$ denotes the $n$-fold convolution of $H$.

For $F \in P_{\mathbb{R}_{+}}$and $r \in R_{+}$, we define the approximation $F_{r}$ by

$$
F_{r}(x)= \begin{cases}F(x) & (0 \leq x<r) \\ 1 . & (x \geq r)\end{cases}
$$

This approximation can be interpreted as the distribution obtained by setting all observations greater than $r$ equal to $r$. The limiting cases $r=0$ and $r=\infty$ correspond to respectively the distribution concentrated in zero and the original distribution $F$.

We shall interpret $\sum_{i=a}^{b} v_{i}=0$ when $b<a$.

\section{RESULTS}

3.1. The following lemma is proved as formula (38) in De Pril \& Dhaene (1992) for the special case $r=1$; the proof is easily extended to the general case.

Lemma 1 For $H \in P_{\mathbb{R}_{+}}, r, m \in Z_{+}$such that $r \leq m$, and $x \in R_{+}$, we have

$$
(m-r) \bar{H}(x) \leq \overline{H^{m *}}(x)-\overline{H^{r *}}(x) \leq(m-r) \mu_{H} .
$$

Lemma 2 For $P \in P_{Z_{+}}, H \in P_{\mathbb{R}_{+}}, r \in Z_{+}$, and $x \in R_{+}$, we have

$$
\bar{H}(x) \bar{P}(r) \leq \overline{P \vee H}(x)-\overline{P_{r} \vee H}(x) \leq \mu_{H} \bar{P}(r) .
$$


Proof. We have

$$
\left.\overline{P \vee H}(x)-\overline{P_{r} \vee H}(x)=\sum_{n=r}^{\infty} p(n) \overline{\left(H^{n *}\right.}(x)-\overline{H^{r *}}(x)\right) .
$$

Application of Lemma 1 gives

$$
\sum_{n=r}^{\infty} p(n)(n-r) \bar{H}(x) \leq \overline{P \vee H}(x)-\overline{P_{r} \vee H}(x) \leq \sum_{n=r}^{\infty} p(n)(n-r) \mu_{H},
$$

from which we obtain (1).

Q.E.D

The second inequality in (1) was proved under more general assumptions by Sundt (1991), who also showed that $0 \leq \overline{P \vee H}(x)-\overline{P_{r} \vee H}(x)$, which is weaker than the first inequality in (1).

If $P_{r}=P$, that is, $P(\mathrm{r})=1$, then the bounds in (1) become equal to zero.

Lemma 1 appears as a special case of Lemma 2 by letting $P$ be the distribution concentrated in $m$.

3.2. For $P, Q \in P_{Z_{+}}, H \in P_{R_{+}}, r \in Z_{+}$, and $x \in R_{+}$, we introduce

$$
B_{r}(x ; P, Q, H)=\overline{P_{r} \vee H}(x)-\overline{Q_{r} \vee H}(x)+\mu_{H} \bar{P}(r)-\bar{H}(x) \bar{Q}(r),
$$

which can also be written as

$$
\begin{aligned}
& B_{r}(x ; P, Q, H)=\sum_{n=1}^{r-1}(p(n)-q(n)) \overline{H^{n *}}(x)- \\
& \left(P(r-1)-Q(r-1) \overline{H^{r *}}(x)+\mu_{H} \bar{P}(r)-\bar{H}(x) \bar{Q}(r) .\right.
\end{aligned}
$$

Theorem 1 For $P, Q \in \mathcal{P}_{Z_{+}}, H \in \mathcal{P}_{\mathbb{R}_{+}}, r \in Z_{+}$, and $x \in R_{+}$, we have

$$
-B_{r}(x ; Q, P, H) \leq \overline{P \vee H}(x)-\overline{Q \vee H}(x) \leq B_{r}(x ; P, Q, H) .
$$

Proof. Application of Lemma 2 gives

$$
\begin{aligned}
& \overline{P \vee H}(x)-\overline{Q \vee H}(x) \leq \overline{P_{r} \vee H}(x)+\mu_{H} \bar{P}(r)-\overline{Q_{r} \vee H}(x)-\bar{H}(x) \bar{Q}(r)= \\
& B_{r}(x ; P, Q, H),
\end{aligned}
$$

which proves the second inequality in (4). The first inequality follows by symmetry.

This completes the proof of Theorem 1.

Q.E.D.

We shall look at some special cases of Theorem 1:

1. As

$$
B_{r}\left(x ; P, P_{r}, H\right)=\mu_{H} \bar{P}(r) \quad B_{r}\left(x ; P_{r}, P, H\right)=-\bar{H}(x) \bar{P}(r),
$$

we see that Lemma 2 (and thus also Lemma 1) is a special case of Theorem 1. 
2. From (3) we obtain

$$
\begin{aligned}
& B_{1}(x ; P, Q, H)=-(p(0)-q(0)) \bar{H}(x)+\mu_{H} \bar{P}(1)-\bar{H}(x) \bar{Q}(1)= \\
& \left(\mu_{H}-\bar{H}(x)\right) \bar{P}(1)+\bar{H}(x)\left(\mu_{P}-\mu_{Q}\right) .
\end{aligned}
$$

For $H \in \mathcal{P}_{Z_{+}}$this case is discussed in Dhaene \& Sundt (1996).

3. From (2) we obtain

$$
B_{0}(x ; P, Q, H)=\mu_{H} \mu_{P}-\bar{H}(x) \mu_{Q} .
$$

4. If $P(r)=Q(r)=1$, then $P_{r}=P$ and $Q_{r}=Q$, and from (2) we obtain

$$
B_{r}(x ; P, Q, H)=-B_{r}(x ; Q, P, H)=\overline{P \vee H}(x)-\overline{Q \vee H}(x),
$$

that is, in this case Theorem 1 becomes trivial.

5. From (2) we obtain

$$
B_{r}(x ; P, P, H)=\left(\mu_{H}-\bar{H}(x)\right) \bar{P}(r),
$$

that is, unfortunately the bounds in Theorem 1 do not in general become equal to zero when comparing two identical compound distributions.

3.3. Let $D_{r}(x ; P, Q, H)$ denote the difference between the upper and lower bound in Theorem 1, that is,

$$
D_{r}(x ; P, Q, H)=B_{r}(x ; P, Q, H)+B_{r}(x ; Q, P, H) .
$$

Then

$$
D_{r}(x ; P, Q, H)=\left(\mu_{H}-\bar{H}(x)\right)(\bar{P}(r)+\bar{Q}(r)) .
$$

We see that $D_{r}(x ; P, Q, H)$ decreases to zero when $r$ increases to infinity, that is, we can make the difference between the upper and lower bound in Theorem 1 as small as desired by making $r$ sufficiently large.

We see that $D_{r}(x ; P, Q, H)$ increases from zero to $\mu_{H}(\bar{P}(r)+\bar{Q}(r))$ when $x$ increases from zero to infinity. Thus our bounds are most accurate for low values of $x$. Furthermore, if for some $\varepsilon>0$ we choose $r$ such that

$$
\bar{P}(r)+\bar{Q}(r)<\frac{\varepsilon}{\mu_{H}},
$$

then $D_{r}(x ; P, Q, H)<\varepsilon$ for all $x \in R_{+}$.

3.4. Let

$$
b_{r}(x ; P, Q, H)=B_{r}(x ; P, Q, H)-B_{r+1}(x ; P, Q, H)
$$


From (3) and trivial calculus we obtain

$$
\begin{aligned}
& b_{r}(x ; P, Q, H)=(P(r)-Q(r))\left(\overline{H^{(r+1) *}}(x)-\overline{H^{r *}}(x)\right)+ \\
& \mu_{H}(1-P(r))-\bar{H}(x)(1-Q(r)) .
\end{aligned}
$$

By rewriting (9) as

$$
\begin{aligned}
& b_{r}(x ; P, Q, H)=(1-P(r))\left[\mu_{h}+\overline{H^{r *}}(x)-\overline{H^{(r+1) *}}(x)\right]+ \\
& (1-Q(r))\left[\overline{H^{(r+1)^{*}}}(x)-\overline{H^{r *}}(x)-\bar{H}(x)\right]
\end{aligned}
$$

and application of Lemma 1 , we see that $b_{r}(x ; P, Q, H) \geq 0$. Thus $B_{r}(x ; P, Q, H)$ is nonincreasing in $r$. This implies that in (4), the upper bound is non-increasing and the lower bound is non-decreasing in $r$, and as $D_{r}(x ; P, Q, H)$ goes to zero when $r$ goes to infinity, both bounds converge to $\overline{P \vee H}(x)-\overline{Q \vee H}(x)$.

Formula (9) can be applied for recursive evaluation of $B_{r}(x ; P, Q, H)$.

Furthermore, when we have found $B_{r}(x ; P, Q, H)$, we easily obtain $B_{r}(x ; Q, P, H)$ from (7) and (8).

3.5. The main purpose of the present subsection is to deduce an improvement of the bounds in Theorem 1. For doing that, we shall need the following lemma.

Lemma 3 For $P, Q \in P_{Z_{+}}, H \in P_{R_{+}}, r \in Z_{+}$and $x \in R_{+}$, we have

$$
b_{r}(x ; P, Q, H) \geq\left(\mu_{H}-\bar{H}(x)\right)(1-\max (P(r), Q(r))) .
$$

Proof. We apply Lemma 1 in (9). If $P(r) \geq Q(r)$, then

$$
b_{r}(x ; P, Q, H) \geq(P(r)-Q(r)) \bar{H}(x)+\mu_{H}(1-P(r))-\bar{H}(x)(1-Q(r)),
$$

that is,

$$
b_{r}(x ; P, Q, H) \geq\left(\mu_{H}-\bar{H}(x)\right)(1-P(r)) .
$$

Analogously, if $P(r)<Q(r)$, then

$$
b_{r}(x ; P, Q, H) \geq(P(r)-Q(r)) \mu_{H}+\mu_{H}(1-P(r))-\bar{H}(x)(1-Q(r)),
$$

that is,

$$
b_{r}(x ; P, Q, H) \geq\left(\mu_{h}-\bar{H}(x)\right)(1-Q(r)) .
$$

From (11) and (12) we obtain (10). 
Theorem 2 For $P, Q \in P_{Z_{+}}, H \in P_{R_{+}}, r \in Z_{+}$, and $x \in R_{+}$, we have

$$
\begin{aligned}
& B_{r}(x ; Q, P, H)+\left(\mu_{H}-\bar{H}(x)\right) \sum_{k=r}^{\infty}(1-\max (P(k), Q(k))) \leq \\
& \overline{P \vee H}(x)-\overline{Q \vee H}(x) \leq \\
& B_{r}(x ; P, Q, H)-\left(\mu_{H}-\bar{H}(x)\right) \sum_{k=r}^{\infty}(1-\max (P(k), Q(k))) .
\end{aligned}
$$

Proof. For $s \in Z_{+}$such that $s \geq r$, we obtain by applying successively Theorem 1 and Lemma 3

$$
\begin{aligned}
& \overline{P \vee H}(x)-\overline{Q \vee \bar{H}}(x) \leq B_{s}(x ; P, Q, H)= \\
& B_{r}(x ; P, Q, H)-\sum_{k=r}^{s-1} b_{k}(x ; P, Q, H) \leq \\
& B_{r}(x ; P, Q, H)-\left(\mu_{H}-\bar{H}(x)\right) \sum_{k=r}^{s-1}(1-\max (P(k), Q(k))) .
\end{aligned}
$$

By letting $s$ go to infinity, we obtain the second inequality in (13); the first inequality follows by symmetry.

This completes the proof of Theorem 2.

Q.E.D.

We see that in (13), like in (4), the lower bound is non-decreasing in $r$ and the upper bound is non-increasing in $r$.

The infinite summation in (13) may seem complicated. However, as all the terms are non-negative, we obtain weaker bounds by including only a finite number of terms. Furthermore, if $P$ or $Q$ has a finite support, then only a finite number of terms are non-zero.

In the following corollary we consider another case where the summation obtains a particularly simple form.

Corollary 1 Let $P, Q \in \mathcal{P}_{Z_{+}}, H \in \mathcal{P}_{\mathbb{R}_{+}}$, and $x \in \mathbb{R}_{+}$. If there exists a non-negative integer $s$ (possibly equal to infinity) such that

$$
\left.\begin{array}{ll}
Q(y) \leq P(y) & (y=0,1, \ldots, s-1) \\
Q(y) \geq P(y), & (y=s, s+1, \ldots)
\end{array}\right\}
$$

then

$$
\begin{array}{r}
-B_{r}(x ; Q, P, H)+\left(\mu_{H}-\bar{H}(x)\right)(\bar{P}(r)-\bar{P}(s)+\bar{Q}(s)) \leq \\
\overline{P \vee H}(x)-\overline{Q \vee H}(x) \leq \\
B_{r}(x ; P, Q, H)-\left(\mu_{H}-\bar{H}(x)\right)(\bar{P}(r)-\bar{P}(s)+\bar{Q}(s)) \\
(r=0,1, \ldots, s-1)
\end{array}
$$




$$
\begin{array}{lr}
-B_{r}(x ; Q, P, H)+\left(\mu_{H}-\bar{H}(x)\right) \bar{Q}(r) \leq \overline{P \vee H}(x)-\overline{Q \vee H}(x) \leq \\
B r(x ; P, Q, H)-\left(\mu_{H}-\bar{H}(x)\right) \bar{Q}(r) . & (r=s, s+1, \ldots)
\end{array}
$$

Proof. For $r=0,1, \ldots, s-1$, we have

$$
\begin{aligned}
& \sum_{k=r}^{\infty}(1-\max (P(k), Q(k)))=\sum_{k=r}^{s-1}(1-P(k))+\sum_{k=s}^{\infty}(1-Q(k))= \\
& \bar{P}(r)-\bar{P}(s)+\bar{Q}(s),
\end{aligned}
$$

and insertion in (13) gives (15). The inequalities (16) are proved analogously.

This completes the proof of Corollary 1 .

Q.E.D.

If we in addition to (14) assume that $\mu_{Q} \leq \mu_{P}$, then we have the stop-loss orderings $\bar{Q} \leq \bar{P}$ and $\overline{Q \vee H} \leq \overline{P \vee H}$; for proofs cf. e.g. Goovaerts, Kaas, van Heerwaarden, \& Bauwelinckx (1990).

At the end of subsection 3.2. we pointed out that unfortunately the bounds in Theorem 1 do not become equal to zero when $Q=P$. From (6) and (15) we see that the improved bounds of Theorem 2 do not have this deficiency.

\section{ACKNOWLEDGEMENT}

The authors are grateful to an anonymous referee for useful suggestions.

\section{REFERENCES}

De PriL, N. \& DhaEne, J. (1992). Error bounds for compound Poisson approximations to the individual risk model. ASTIN Bulletin 22, 135-148.

DhAENE, J. \& SUNDT, B. (1996). On error bounds for approximations to aggregate claims distributions. Submitted for publication in ASTIN Bulletin.

Goovaerts M.J., KaAs, R., van Heerwaarden, A.E., \& Bauwelinckx, T. (1990). Effective Actuarial Methods. North-Holland, Amsterdam.

SunDT, B. (1991). On approximating aggregate claims distributions and stop-loss premiums by truncation. Insurance: Mathematics and Economics 10, 133-136.

BJøRN SUNDT

Department of Mathematics

University of Bergen

Allégaten 55

$N$-5007 Bergen

Norway
JAN DHAENE

Departement Toegepaste Economische Wetenschappen

Katholieke Universiteit Leuven

Huis Eighen Heerd

Minderbroederstraat 5

B-3000 Leuven

Belgium 